
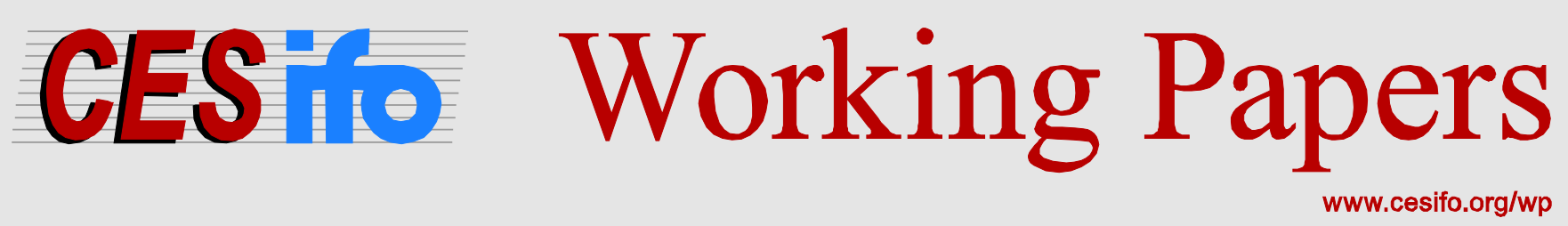

\title{
Modeling Damages in Climate Policy Models: Temperature-Based or Carbon-Based?
}

\author{
Charles F. Mason \\ Neil Wilmot
}

\author{
CESIFO WORKING PAPER NO. 5287 \\ CATEGORY 10: ENERGY AND CLIMATE ECONOMICS \\ MARCH 2015
}

An electronic version of the paper may be downloaded

- from the SSRN website:

- from the RePEc website:

- from the CESifo website:

www.SSRN.com

Www.RePEc.org

www.CESifo-group.org/wp 


\title{
Modeling Damages in Climate Policy Models: Temperature-Based or Carbon-Based?
}

\begin{abstract}
Economists have analyzed potential for damages from climate change from theoretical analyses and with Integrated Assessment Models (IAMs). Analytical models typically write damages as a function of the carbon stock, while IAMs typically view damages as based on temperatures. In this paper, we evaluate the implications for adapting analytic models to include two state variables - temperature and carbon stocks. We first provide an analytical comparison of a model where damages are based on carbon stocks against a model where damages are based on temperatures. When damages are based on carbon stocks, the time path of optimal emissions is described by a first-order differential equation; when damages are based on temperatures, the time path of optimal emissions is described by a second-order differential equation. We then proceed to an empirical analysis of the link between temperatures and carbon stocks. Our empirical analysis strongly supports a relation between levels of carbon stocks and changes in temperatures, and indicates the virtual absence of a linkage between levels of carbon and levels of temperature. As such, it is broadly supportive of a more elaborate modeling structure, under which two state variables are included in the analytical framework.
\end{abstract}

Keywords: climate change, damage function.

Charles F. Mason

H.A. True Chair in Petroleum and Natural

Gas Economics / Department of Economics

\& Finance, University of Wyoming

1000 E. University Ave.

USA - Laramie, WY 82071

bambuzlr@uwyo.edu
Neil Wilmot

Department of Economics

University of Minnesota-Duluth

1318 Kirby Drive

USA - Duluth, MN 55812-3029

nwilmot@d.umn.edu

December 23, 2014

We thank, without implicating, participants at the 2014 CESifo Area Conference on Energy and Climate Economics for helpful discussion. 


\section{Introduction}

Perhaps the most pressing issue currently facing environmental economists is the potential large-scale damages that might obtain from climate change. This problem has been investigated from a variety of perspectives, from purely theoretical analyses to detailed and sophisticated numerical simulations (often called "Integrated Assessment Models," or IAMs). Aside from the methodological differences in these two approaches, they often differ in certain key modeling assumptions. Of particular interest is the way that damages are modeled. Analytical models typically write damages asa function of the carbon stock, while IAMs typically view damages as based on temperatures, and build in a numerical algorithm to link carbon stocks to temperature changes. Examples of the former include Chakravorty et al. (2006); Dutta and Radner (2009); Forster (1980); Grimaud and Rouge (2014); Harstad (2012); Jouvet et al. (2005); Karp and Zhang (2006); Michel and Rotillon (1995); van der Ploeg and Withagen (2014); Tahvonen (1997) and Ulph and Ulph (1997), while examples of the latter include Crost and Traeger (2013); Golosov et al. (2014); Kelly and Kolstad (1999); Leach (2007); Lemon and Traeger (2014); Nordhaus (2008) and Pizer (1999).

It seems clear that the principal causal factor in damages will be the climate itself, perhaps proxied by some measure of temperature (e.g., global mean temperature). Carbon stocks only matter to the extent they influence the evolution of climate. For damages to be linked to Carbon stocks, one must implicitly assume that the linkage from Carbon to temperatures is direct, with levels in carbon impacting temperature levels. But there are 
physical reasons that changes in, not levels of, global temperatures are impacted by radiative forcing, which in turn is linked to carbon stock (Allen et al., 2009). Taken literally, these facts imply that one must go beyond a simple one-state-variable model, allowing instead for two state variables-temperature and carbon stocks. But this extra complication does impose a modeling cost, in that it renders analyses more complex, and has the potential to obscure interpretations. As such, it seems prudent to evaluate the distinction between an analytical model that bases damages on carbon stocks and an analytical model that bases damages on temperature. Our goal in this paper is to provide such an evaluation.

We start our investigation by providing a continuous-time dynamic optimization model of climate policy in Section 2. Here we compare a structure where damages are based on carbon stocks to a structure where damages are based on temperatures; a specific comparison is offered for two representations that have received considerable attention in the literature, namely where damages are additive (and so are subtracted from the gross flow of benefits) and one where damages are multiplicative (and so scale down benefits). In Section 3, we compare the two modeling structures; this boils down to a comparison between the differential equations that describe the time path of optimal emissions. When damages are based on carbon stocks, the time path of optimal emissions is described by a first-order differential equation; when damages are based on temperatures, the time path of optimal emissions is described by a second-order differential equation. We then proceed to an empirical analysis of the link between temperatures and carbon stocks, in Section 4. Using readily available data on carbon stocks, global emissions, and global temperatures we 
evaluate the relations underpinnings economic analyses. The empirical analysis strongly supports a relation between levels of carbon stocks and changes in temperatures, and indicates the virtual absence of a linkage between levels of carbon and levels of temperature. As such, it is broadly supportive of a more elaborate modeling structure, under which two state variables are included in the analytical framework. We offer concluding comments in Section 5.

\section{Conceptual Underpinnings}

A number of authors have investigated the dynamic optimization problem associated with climate change. By and large, the typical analytical investigation models damages as based on carbon stocks. ${ }^{1}$ In such a setting, the key state variable is the stock of atmospheric carbon, $C$. The evolution of this state variable is commonly expressed as the difference between the flow of (unabated) emissions, $E$, and the rate of natural assimilation of carbon into natural sinks, $f(C)$ :

$$
\dot{C}=E-f(C) \text {. }
$$

While it is analytically convenient to assume natural uptake is linear in the carbon stock, which corresponds to exponential decay, Solomon et al. (n.d.) has noted that some carbon stays in the atmosphere for a very long time. Thus, assuming exponential decay of the

1 Indeed, damages are often assumed to take a simple functional form, e.g.quadratic. Basing damages on carbon stocks is a seemingly natural application of stock-related damages, in that current economic activity, represented by the flow of emissions, maps naturally into changes in atmospheric carbon. This modeling approach has a long history, going back at least to Forster (1980). 
(complete) carbon stock is incompatible with physical reality. Allen et al. (2009) offer a more satisfactory physical model, based on multiple carbon stocks that reflect different time scales. For example, writing long-term equilibrium carbon stocks as $C_{3}$ and deviations away from those stocks as $C_{2}$, the system satisfies

$$
\begin{aligned}
& \dot{C}_{2}=a_{0} E-b_{2} C_{2} ; \\
& \dot{C}_{3}=b_{3} C_{3} .
\end{aligned}
$$

An alternative scenario, that does not require multiple state variables to measure carbon, would be to allow for non-linear decay. In such a model, the uptake function $f(C)$ is increasing but concave in the carbon stock: $f^{\prime}(C)>0, f^{\prime \prime}(C)<0$. We will adopt this modeling convention in the pursuant discussion. We start by investigating a simple model wherein damages are tied to atmospheric carbon, and then proceed to a more realistic model wherein temperatures determine damages.

\subsection{Damages as a Function of Carbon}

To analyze the significance of characterizing damages as a function of temperature, we start by investigating the simplified model where damages depend only on the stock of carbon. In this setting, we write payoffs at any point in time $t$ as $U(E, C)$. These payoffs could be additive in damages, in which case $U(E, C)=u(E)-\Delta(C)$, where $u$ would be interpreted as net benefits from unabated emissions, for example GDP net of sequestration and abate- 
ment costs, and $\Delta(C)$ would be damages. Alternatively, payoffs could be multiplicative in damages, with $U(E, C)=\delta(C) u(E)$, where $1-\delta(C)$ is interpreted as the reduction in net benefits associated with damages. For either variation, it is natural to assume the payoff function is increasing at small levels of $E$ and globally concave.

The social planner's problem is to select a time path of unabated emissions that maximizes the present discounted flow of payoffs:

$$
\begin{aligned}
\max _{E_{t}} & \int_{0}^{\infty} U\left(E_{t}, C_{t}\right) e^{-\rho t} d t \\
& \text { subject to: } \quad \dot{C}_{t}=E_{t}-f\left(C_{t}\right) ;
\end{aligned}
$$

where $\rho$ is the discount rate.

The solution to this problem is described by Pontryagin's maximum principle: we first define the current-value Hamiltonian

$$
\mathcal{H}=U(E, C)+m[E-f(C)]
$$

where $m$ is the co-state variable (i.e., shadow value) associated with the state variable $C$. The optimal level of emissions maximizes the Hamiltonian at each point in time; assuming interior solutions, we have

$$
\frac{\partial U}{\partial E}+m=0
$$


The solution also requires that the co-state variable follows the equation of motion

$$
\begin{aligned}
\dot{m} & =\rho m-\frac{\partial \mathcal{H}}{\partial C} \\
& =\left[\rho+f^{\prime}(C)\right] m-\frac{\partial U}{\partial C} .
\end{aligned}
$$

To make additional headway, we time-differentiate equation (3) to obtain

$$
\left(\frac{\partial^{2} U}{\partial E^{2}}\right) \dot{E}+\left(\frac{\partial^{2} U}{\partial E \partial C}\right) \dot{C}+\dot{m}=0
$$

Using eqs. (3), (4) and (5), and combining terms, we obtain

$$
\dot{E}=E\left[\rho+f^{\prime}(C)\right] \varepsilon_{E}+\frac{\partial U / \partial C}{\partial^{2} U / \partial E^{2}}-(E-f(C))\left[\frac{\partial^{2} U}{\partial E \partial C} / \frac{\partial^{2} U}{\partial E^{2}}\right]
$$

where $\varepsilon_{E}$ is the elasticity of marginal payoffs with respect to emissions, $\frac{\partial U}{\partial E} /\left(E \frac{\partial^{2} U}{\partial E^{2}}\right)$.

Combining equation (6) with the state equation governing the carbon stock generates a system of two first-order differential equations. The solution to this system then yields the time path of emissions and the carbon stock.

\subsection{Damages as a Function of Temperatures}

Linking damages to carbon stocks would make sense if temperatures were directly related to carbon stocks, through a monotonic transformation $T=\phi(C)$. In the absence of such a transformation, the dynamic optimization problem associated with framing optimal climate 
policy would need to include at least two state variable, temperatures and carbon stocks.

Such a model can be constructed starting from Weitzman (2010). Let $T(t)$ represent a measure of temperature - such as global mean temperature - at time $t$, and denote the stock of atmospheric carbon by $C(t)$. The equation of motion for temperature can then be written as (Allen et al., 2009; Weitzman, 2010):

$$
\dot{T}=\alpha \ln \left(C / C_{0}\right)-\beta T,
$$

where $C_{0}$ measures pre-industrial atmospheric levels of $\mathrm{CO}_{2}$.

At any point in time $t$ payoffs are $\Pi(E, T)$. These payoffs could be additive in damages, in which case $\Pi(E, T)=\pi(E)-D(T)$, where $\pi$ would be interpreted as net benefits from unabated emissions, for example GDP net of sequestration and abatement costs, and $D$ would be temperature-related damages. Alternatively, payoffs could be multiplicative in damages, with $\Pi(E, T)=d(T) \pi(E)$, where $1-d(T)$ is interpreted as the reduction in net benefits associated with temperature-related damages. It is natural to assume the payoff function is increasing at small levels of $E$ and globally concave.

The social planner's problem is to select a time path of unabated emissions that maxi- 
mizes the present discounted flow of payoffs:

$$
\begin{aligned}
\max _{E_{t}} & \int_{0}^{\infty} \Pi\left(E_{t}, T_{t}\right) e^{-\rho t} d t \\
\text { subject to: } & \dot{C}_{t}=E_{t}-f\left(C_{t}\right), \\
\dot{T} & =\alpha \ln \left(C / C_{0}\right)-\beta T .
\end{aligned}
$$

The solution to this problem is described by Pontryagin's maximum principle: we first define the current-value Hamiltonian

$$
\mathcal{H}=\Pi(E, T)+\mu[E-f(C)]+v\left[\alpha \ln \left(C / C_{0}\right)-\beta T\right],
$$

where $\mu$ and $\nu$ refer to the co-state variables (i.e., shadow values) associated with the state variables $C$ and $T$, respectively. The optimal values of the controls maximize the Hamiltonian at each point in time; assuming interior solutions, we therefore have

$$
\frac{\partial \Pi}{\partial E}+\mu=0
$$


The solution also requires the co-state variables follow the equations of motion

$$
\begin{aligned}
\dot{\mu} & =\rho \mu-\frac{\partial \mathcal{H}}{\partial C} \\
& =\left[\rho+f^{\prime}(C)\right] \mu-\frac{\alpha \nu}{C} ; \\
\dot{v} & =\rho \nu-\frac{\partial \mathcal{H}}{\partial T} \\
& =[\rho+\beta] \nu-\frac{\partial \Pi}{\partial T} .
\end{aligned}
$$

To make additional headway, we time-differentiate equation (9) to obtain

$$
\left(\frac{\partial^{2} \Pi}{\partial E^{2}}\right) \dot{E}+\left(\frac{\partial^{2} \Pi}{\partial E \partial T}\right) \dot{T}+\dot{\mu}=0
$$

Using eqs. (9), (10) and (12), and combining terms, we obtain

$$
\dot{E}=E\left[\rho+f^{\prime}(C)\right] \sigma_{E}+\left(\frac{\alpha}{C \partial^{2} \Pi / \partial E^{2}}\right) v-\left[\alpha \ln \left(C / C_{0}\right)-\beta T\right]\left(\frac{\partial^{2} \Pi}{\partial E \partial T} / \frac{\partial^{2} \Pi}{\partial E^{2}}\right)
$$

where $\sigma_{E}$ is the elasticity of marginal payoffs with respect to emissions, $\frac{\partial \Pi}{\partial E} /\left(E \frac{\partial^{2} \Pi}{\partial E^{2}}\right)$. The key points to be gleaned from equations (9) and (13) is that damages only exert an indirect effect on optimal emissions, in that they influence changes in - but not levels of - optimal emissions. That is, the role played by climate change in determining optimal policy at a point in time is far subtler than would be suggested by a simpler model that regards damages as the result of the stock of greenhouse gases. 


\section{Comparing the Two Models}

Combining equation (13) with the state equations governing the carbon stock and temperature generates a system of three first-order differential equations. The solution to this system then yields the time path of emissions, the carbon stock and temperatures. We note that one could reduce this system by time-differentiating equation (13), and then using the state equation of $T$ to eliminate temperature from the system. In this way, a second-order differential equation for emissions can be linked to global carbon stock. This characterization is plainly different from the system of two first-order differential equations one would obtain in the context of the simpler model where damages are linked to carbon stocks.

To facilitate a more in-depth comparison, we investigate two specific formulations of payoffs that have been considered in the literature: one where damages are additive, and one where damages are multiplicative. In both representations, we will assume that gross payoffs associated with the flow of emissions are iso-elastic.

\subsection{Additive Damages}

We first analyze a version of the model where utility is comprised of payoffs from consumption, which is tied to emissions, minus the damages incurred as a result of the relevant state variable. In such a version of the model, we have $U(E, C)=u(E)-D(C)$ in the context of subsection 2.1 and $\Pi(E, T)=\pi(E)-\Delta(T)$ in the context of subsection 2.2. We assume 
the flow of utility payment functions are equal, and are iso-elastic: $u(E)=\pi(E)=E^{\gamma}$; then

$$
\frac{u^{\prime}(E)}{E u^{\prime \prime}(E)}=\frac{1}{\gamma-1}=\frac{\pi^{\prime}(E)}{E \pi^{\prime \prime}(E)} .
$$

The equation of motion governing optimal emissions in subsection 2.1 can be rewritten as

$$
\dot{E}=\left[\rho+f^{\prime}(C)-\frac{E D^{\prime}(C)}{u^{\prime}(E)}\right]\left(\frac{E}{\gamma-1}\right) .
$$

The equation of motion governing optimal emissions in subsection 2.2 can be rewritten as

$$
\dot{E}=\left[\rho+f^{\prime}(C)+\left(\frac{\alpha}{\pi^{\prime}(E)}\right)\left(\frac{E}{C}\right) \vee\right]\left(\frac{E}{\gamma-1}\right) \text {. }
$$

Inspecting eqs. (14) and (15), and recalling that $u(E)=\pi(E)$, we see that the difference boils down to a comparison of $\alpha v$ with $-C D^{\prime}(C)$. In light of the evolution of the shadow value $v$, it is apparent that these terms are unlikely to coincide except by accident. In other words, basing damages on carbon stocks will lead to approximation errors that change over time.

\subsection{Multiplicative Damages}

We next analyze a version of the model where damages are multiplicative. In this variant, payoffs are proportional to a factor that reflects the the damages incurred as a result of the relevant state variable. In the context of subsection 2.1, we have $U(E, C)=\delta(C) u(E)$. 
In the context of subsection 2.2, we have $\Pi(E, T)=d(T) \pi(E)$. As in the preceding subsection, we assume $u(E)=\pi(E)=E^{\gamma}$.

The equation of motion governing optimal emissions in subsection 2.1 can be rewritten as

$$
\dot{E}=\left[\rho+f^{\prime}(C)-\frac{E \Delta^{\prime}(C)}{u^{\prime}(E)}+\delta^{\prime}(C)[f(C)-E]\right]\left(\frac{E}{\gamma-1}\right) .
$$

The equation of motion governing optimal emissions in subsection 2.2 can be rewritten as

$$
\dot{E}=\left[\rho+f^{\prime}(C)+\left(\frac{\alpha}{\pi^{\prime}(E)}\right)\left(\frac{E}{C}\right) v-d^{\prime}(T)\left[\alpha \ln \left(C / C_{0}\right)-\beta T\right]\right]\left(\frac{E}{\gamma-1}\right) .
$$

Inspecting eqs. (16) and (17), and recalling that $u(E)=\pi(E)$, we see that a comparison of the equations of motion on emissions involves a comparison of $\alpha v / C$ with $d^{\prime}(C)$. But the distinction between the terms $d^{\prime}(T) \dot{T}$ and $\delta^{\prime}(C) \dot{C}$ also enters. Were there a monotonic transformation $T=\phi(C)$ that tied temperature levels to carbon stocks, then $\dot{T}=\phi^{\prime}(C) \dot{C}$ and so $\delta^{\prime}(C) \dot{C}=d^{\prime}(T) \phi^{\prime}(C) \dot{T} / \phi^{\prime}(C)=d^{\prime}(T) \dot{T}$. As we noted above, however, there are reasons to doubt the existence of such a monotonic transformation. As in the preceding sub-section, then, we conclude that the time path of emissions under a model with damages based on carbon stocks would only coincide with the time path of emissions under a model with damages based on carbon stocks by accident. Here again, basing damages on carbon stocks will lead to approximation errors that change over time.

The main message from this analysis is that adopting the simplifying assumption that damages are based on carbon stocks, as opposed to temperatures, is likely to induce ap- 
proximation errors unless there is a monotonic transformation that links temperature levels to carbon stocks. In the next section, we investigate the empirical plausibility of such a monotonic transformation.

\section{Empirical Analysis of Temperatures}

The preceding analysis provides a conceptual framework for comparing models where damages are linked to carbon stocks with models where damages are linked to temperature. At its core, the key distinction between these models boils down to the state equation governing temperature stocks, eq. (7). In essence, the role played by carbon stocks is intermediate if they impact the rate of change in temperatures, whereas the role played by carbon stocks can be viewed as primary if they impact the level of temperatures. Putting these comparisons on the same footing, the question boils down to this: is it levels of carbon, or changes in carbon, that impact the change in temperature? In this section, we provide empirical evidence on this comparison.

\subsection{Data and methodology}

To shed light on this issue, we collected data on global mean temperatures and atmospheric carbon. ${ }^{2}$ Carbon stocks are measured at a number of locations. We use observations from Mauna Loa, which represent the longest time series of atmospheric carbon observations.

\footnotetext{
2 Atmospheric carbon data are available at ftp://aftp.cmdl.noaa.gov/products/trends/co2/co2_mm_mlo.txt. Global mean temperature data are available at http://data.giss.nasa.gov/gistemp/tabledata/GLB.Ts+dSST.txt.
} 
Data on both global mean temperature and carbon stocks are available at the monthly level from March 1958 to August 2014, which yields 678 observations.

Using this data, we investigate eq. (7). The regression equation of interest is:

$$
\Delta T_{t}=\beta_{0}+\beta_{1} \ln C_{t}+\beta_{2} T_{t}+\varepsilon_{t},
$$

where $T_{t}$ is global mean temperature at time $t, \Delta T_{t}$ is the change in global mean temperature, $T_{t}-T_{t-1}, \ln C_{t}$ is the natural $\log$ of atmospheric carbon at time $t, \varepsilon_{t}$ is the disturbance term at time $t$, and $\beta_{0}, \beta_{1}, \beta_{2}$ are parameters to be estimated. The hypothesis of interest is that $\beta_{1}>0$.

Equation (18) could equally well be viewed as one where the left-side variable is current temperature, in which case one of the regressors is the lagged value of the left-side variable. This fact poses no difficulties if the residual term is serially uncorrelated, but that seems suspect in the case at hand. Because temperatures evolve slowly, the potential for shocks in one month to exert an influence on temperatures in the next month would seem to be very real. Accordingly, we proceed on the assumption that we have serial correlation as well as a lagged left-side variable in the regression equation. Addressing this complication requires the procurement of a consistent estimate of the serial correlation parameter; we follow the approach suggested by Hamilton (1994, p. 226). ${ }^{3}$ We will also consider a variation on the

3 The idea is to run a regression that adds lagged values of the original regressors, and then form the negative of the ratio of the point estimates on current and lagged values of the exogenous variable (the natural $\log$ of carbon stock). This point estimate is a consistent estimator of the true underlying serial correlation parameter. We then transform the variables by subtracting the product of this estimate with the lagged value of the variable, for each of our variables. The point estimates reported below are derived from such a transformation. Because this approach requires dropping the first two observations, the regressions employ 676 
regression equation that includes the change in atmospheric carbon, $\Delta C_{t}=C_{t}-C_{t-1}$, as a regressor; the simple model that uses carbon as the only state variable can be interpreted as the hypothesis that the coefficient on $\Delta C_{t}$ is positive.

\subsection{Main results}

Results from regression analysis based on the above model are presented in Table 1. The the second column (labeled 'Regression 1') is based on equation (18); both lagged temperature (measured in degrees Centigrade) and the natural log of atmospheric carbon stocks (measured in parts per million) are strongly statistically significant, and take the anticipated sign. We also note that there is considerable residual uncertainty, as indicated by the modest R-squared value. An alternative regression, that allows for the possible influence of the change in carbon stocks upon the change in temperatures, is reported in the third column ('Regression 2'). We note that the coefficients on lagged temperature and the natural log of atmospheric carbon stocks, as well as the constant, are very similar in the two regressions. Further, the coefficient on the new variable appears insignificant, both numerically and statistically. By contrast, dropping the natural log of carbon stocks, as we do in the fourth column ('Regression 3') yields a noticeable drop in explanatory power (as measured by the sharp reduction in the $R^{2}$ statistic), a noticeable change in the coefficient on lagged temperature, and a sign change in the constant. This third regression corresponds to a model in which there is a direct relation between levels of carbon stock and levels of temperatures, observations. 
as is implied in a number of extant theoretical analyses. In our view, the regression results reported in Table 1 strongly support the physical model described in eq. (7) - where carbon stocks influence changes in temperatures - and cast serious doubt on a model that posits a direct relation between carbon stocks and temperatures.

Overall, these results strongly support the hypothesis that the level of carbon stocks is a key driver in temperature changes, consistent with the representation in the state equation for temperatures, eq. (7). By contrast, there is little evidence to suggest that changes in carbon stocks exert any impact on changes in temperatures, which argues against a direct relation between carbon stocks and temperatures. This aspect of our results argues against the empirical validity of a simple dynamic optimization model that expresses damages in terms of carbon stocks, and that uses only carbon as the sole state variable.

\subsection{Robustness}

There are two possible issues with the analysis undertaken above. The first has to do with the potential endogeneity of carbon stocks, and the second has to do with the time series structure of the error term in our regressions. The concern regarding potential endogeneity of carbon stocks arises because increases in temperatures from one observation to the next, i.e., from one month to the next, can increase vegetation; in turn, this increased vegetation can exert a temproary impact on carbon uptake, which might influence measured carbon stocks. To address this concern, we ran an instrumental variables regression using lagged carbon stocks and lagged carbon dioxide emissions as instruments for current 
carbon stocks. ${ }^{4}$ Lagged carbon stocks and emissions are certainly not caused by current temperatures, while the state equation describing the evolution of carbon stocks suggests there should be a reasonably relation between current stocks on the one hand and lagged stocks and emissions on the other hand. We therefore expect these variables to perform well as instruments for current stock. The results from an instrumental variables regression using these instruments are reported in the fifth column, labeled 'regression 4'. We see here that the coefficients on lagged temperature and the natural log of carbon stocks are quite similar to those in regression 1 , suggesting the potential endogeneity of the carbon stock variable is likely of minimal concern. Finally, we employed a similar instrumental variables approach to re-estimate regression 2, which are listed in the sixth column (labeled 'regression $\left.5^{\prime}\right) .{ }^{5}$ We see again that the coefficients on lagged temperature and the natural $\log$ of carbon stocks are quite similar to those in regression 2, as is the coefficient on the difference in carbon stocks. These results also suggesting the potential endogeneity of the carbon stock variable is likely of minimal concern.

The second issue is that the error structure might be more intricate than our initial analysis admits. One particular possibility is that the system is characterized by time-varying volatility, i.e. that the variance in the error terms changes over time. A general characteri-

4 Data on global $\mathrm{CO}_{2}$ emissions are only available at the annual level, whereas monthly observations on US emissions are available starting in January 1973. We used this set of US monthly emissions to identify the average fraction of annual emissions associated with each of the 12 months, and then used these estimated fractions to construct a synthetic time series of monthly US emissions from March 1958 to December 1972. The corresponding vector can be regarded as an estimate of US monthly emissions. The US was the largest global emitter of $\mathrm{CO}_{2}$ until relatively recently, regularly representing over $20 \%$ of global emissions. Moreover, annual US emissions are closely correlated with global emissions: between 1958 and 2010, the simple correlation between these two time series is $93.4 \%$.

5 Because the inclusion of the change in carbon stocks implies lagged carbon appears as aright-side variable, we use twice lagged carbon stocks and twice lagged emissions as instruments here. 
zation of this phenomenon is the "generalized autoregressive conditional heteroskedastic" (GARCH) framework. Under the GARCH framework, the disturbance term in equation (18) is Normally distributed with mean 0 and variance $\sigma_{t}^{2}$, where:

$$
\sigma_{t}^{2}=\alpha_{0}+\sum_{i=1}^{q} \alpha_{i} \varepsilon_{t-i}^{2}+\sum_{j=1}^{p} \kappa_{i} \sigma_{t-j}^{2}
$$

We refer to this model as the "GARCH(p,q)" model in the pursuant discussion. Note that when $\sigma_{t}^{2}=\sigma^{2}$ the GARCH model reduces to the standard regression model.

The first step in invoking this approach is to determine the appropriate lag lengths ( $p$ and q); we use two criteria that have been proposed in the literature, the "Akaike Information Criterion" (AIC) and the "Bayesian Information Criterion" (BIC) to perform this determination. The idea is to select the combination of $p$ and $q$ that minimizes these criterion. Table 2 provides values of these criteria for a variety of lag combinations. ${ }^{6}$

As is often true in this sort of analysis, the AIC and BIC do not agreee (Hamilton, 1994). In particular, the AIC selects a model with $p=q=4$, while the BIC selects a model with $p=2, q=3$. In light of this ambiguity, we provide estimates under both specifications, in Table 3. The second and third columns list estimates for the model $p=2, q=3$, while the fourth and fifth columns list estimates for the model $p=q=4$. Columns 2 and 4 correspond to column 2 of Table 3, while columns 3 and 5 correspond to column 3 of Table 3 . We observe that the GARCH parameters are jointly statistically significant, pointing to the

6 For some combinations, the numerical algorithm failed to converge. Accordingly, the AIC and BIC are not defined for these combinations, and so we do not list them in Table 2. 
presence of fat tails in the error structure. ${ }^{7}$

The key message from these regressions is that the estimates on both $\ln C_{t}$ and $T_{t-1}$ are quite similar for both variations of both models; these estimates are also close and to the corresponding estimates in Table 1. Moreover, the estimates on $\Delta C_{t}$ in columns 3 and 5 are statistically insignificant, also as in Table 1.

Overall, these results corroborate the results we discussed above. In particular, they are strongly supportive of the role played by the level of carbon stocks as a key driver in temperature changes, but indicate that changes in carbon stocks exert essentially no effect on changes in temperatures. These results are consistent with the representation in the state equation for temperatures, eq. (7).

\section{Conclusion}

In this paper, we consider extensions to the traditional theoretical growth model associated with climate policy, and consider related empirical evidence. Our analytical analysis points to the potential for important modeling errors associated with the use of carbonbased damage functions: in that setting, the time path of optimal emissions is described by a first-order differential equation, whereas the the time path of optimal emissions is

\footnotetext{
7 The maximized value of the log-likelihood function for the $\operatorname{GARCH}(2,3)$ model is $L L^{*}=578.79$, while the maximized value of the log-likelihood function for the model that assumes the error structure is not GARCH is $L L_{0}=561.76$; this yields a test statistic of 34.05 for the null hypothesis the error structure is not GARCH. This test statistic will be distributed as a Chi-squared random variable with 5 degrees of freedom under the null hypothesis. As our test statistic greatly exceeds 15.1 , the $1 \%$ critical value for probability that a Chi-squared random variable with 5 degrees of freedom, we reject the null hypothesis, in favor of the alternative hypothesis that the error structure exhibits GARCH features. (A similar conclusion emerges from a calculation based on a $\operatorname{GARCH}(4,4)$ model).
} 
described by a second-order differential equation when the damage function is based on temperatures. For the assumption that damages are tied to carbon stocks to be plausible, there must be a link between levels of temperatures and levels of carbon (which would then imply a link between changes in carbon and changes in temperature). But our empirical analysis points to a clear link between levels of carbon and changes in temperatures; there is virtually no role for carbon levels to influence temperature levels. Accordingly, our results are strongly supportive of the temperature-based damage representation.

Weitzman (2009) has argued that we need to pay close attention to the relative size of the tails in whatever probability distribution is deemed to characterize the salient uncertainty in modeling optimal climate policy. One aspect of our empirical analysis is supportive of the "fat tail" concept. In modeling the underlying uncertainty that impacts the state equation for temperatures, our robustness analysis points to the likely role played by timevarying volatility, as captured by a "generalized autoregressive conditional heteroskedastic" (GARCH) framework. The GARCH framework implies a kurtosis that is larger than that of a Normal random variable, and so is consistent with the fat tail concept. 


\section{References}

Allen, M. R., Frame, D. J., Huntingford, C., Jones, C. D., Lowe, J. A., Menihausen, M. and Meinhausen, N. (2009). Warming caused by cumulative carbon emissions towards the trillionth tonne, Nature 458: 1163-1166.

Chakravorty, U., Magné, B. and Moreaux, M. (2006). A hotelling model with a ceiling on the stock of pollution, Journal of Economic Dynamics and Control 30: 2875-2904.

Crost, B. and Traeger, C. P. (2013). Optimal climate policy: Uncertainty versus monte carlo, Economics Letters 120: 552-558.

Dutta, P. K. D. and Radner, R. (2009). A strategic analysis of global warming: Theory and some numbers, Journal of Economic Behavior \& Organization 71: 187-209.

Forster, B. A. (1980). Optimal energy use in a polluted environment, Journal of Environmental Economic and Management 7: 321-333.

Golosov, M., Hassler, J., Krusell, P. and Tsyvinski, A. (2014). Optimal taxes on fossil fuel in general equilibrium, Econometrica 82: 41-88.

Grimaud, A. and Rouge, L. (2014). Carbon sequestration, economic policies and growth, Resource and Energy Economics 36: 307-331.

Hamilton, J. D. (1994). Time Series Analysis, 1 edn, Princeton University Press, Princeton, NJ. 
Harstad, B. (2012). Buy coal! a case for supply-side environmental policy, Journal of Political Economy 120: 77-115.

Jouvet, P.-A., Michel, P. and Rotillon, G. (2005). Optimal growth with pollution: how to use pollution permits?, Journal of Economic Dynamics and Control 29: 1597-1609.

Karp, L. and Zhang, J. (2006). Regulation with anticipated learning about environmental damages, Journal of Environmental Economics and Management 51: 259-279.

Kelly, D. L. and Kolstad, C. D. (1999). Bayesian learning, growth, and pollution, Journal of Economic Dynamics and Control 23: 491-518.

Leach, A. J. (2007). The climate change learning curve, Journal of Economic Dynamics and Control 31: 1728-1752.

Lemon, D. and Traeger, C. (2014). Watch your step: Optimal policy in a tipping climate, American Economic Journal: Economic Policy 6: 137-166.

Michel, P. and Rotillon, G. (1995). Disutility of pollution and endogenous growth, Environmental and Resource Economics 6: 279-300.

Nordhaus, W. (2008). A Question of Balance: Weighing the Options on Global Warming Policies, Yale University Press, . New Haven, CT:

Pizer, W. A. (1999). The optimal choice of climate change policy in the presence of uncertainty, Resource and Energy Economics 21: 255-287. 
Solomon, S., Plattner, G.-K., Knutti, R. and Friedlingstein, P. (n.d.). Irreversible climate change due to carbon dioxide emissions, Proceedings of the National Academy of Sciences 106(6): 1704-1709.

Tahvonen, O. (1997). Fossil fuels, stock externalities, and backstop technology, The Canadian Journal of Economics 30: 855-874.

Ulph, A. and Ulph, D. (1997). Global warming, irreversibility and learning, The Economic Journal 107: 636-650.

van der Ploeg, F. and Withagen, C. (2014). Growth, renewables and the optimal carbon tax, International Economic Review 55: 283-311.

Weitzman, M. L. (2009). On modeling and interpreting the economics of catastrophic climate change, Review of Economics and Statistics 91: 1-19.

Weitzman, M. L. (2010). What is the damages function for global warming - and what difference might it make?, Climate Change Economics 1(1): 57-69. 
Table 1: Regression results: global mean temperatures

\begin{tabular}{lccccc}
\hline \hline variable & Regression 1 & Regression 2 & Regression 3 & Regression 4 & Regression 5 \\
\hline$T_{t-1}$ & $-0.2585^{* *}$ & $-0.2589^{* *}$ & $-.0 .0592^{* *}$ & $-0.2579^{* *}$ & $-0.2574^{* *}$ \\
& $(0.0258)$ & $(0.0259)$ & $(0.0172)$ & $(0.0132)$ & $(0.0259)$ \\
$\ln \left(C_{t}\right)$ & $0.8704^{* *}$ & $0.8658^{* *}$ & - & $0.8672^{* *}$ & $0.8655^{* *}$ \\
& $(0.0980)$ & $(0.0984)$ & & $(0.1201)$ & $(0.0985)$ \\
$C_{t}-C_{t-1}$ & - & 0.0096 & 0.0128 & - & 0.0750 \\
& & $(0.0135)$ & $(0.0157)$ & & $(0.2328)$ \\
constant & $-1.766^{* *}$ & $-1.726^{* *}$ & $1.055^{* *}$ & $-1.754^{* *}$ & $-1.748^{* *}$ \\
& $(0.3826)$ & $(0.3867)$ & $(0.2351)$ & $(0.3844)$ & $(0.3844)$ \\
\hline \multirow{2}{*}{$N$} & & & & & 676 \\
$R^{2}$ & 0.130 & 0.129 & 0.029 & 0.129 & 0.129 \\
\hline \hline
\end{tabular}

Dependent variable: $T_{t}-T_{t-1}$

Standard errors in parentheses

*: significant at $5 \%$ level

**: significant at $1 \%$ level 
Table 2: Information criteria for various GARCH lag structures

\begin{tabular}{ccc}
\hline \hline GARCH $(p, q)$ & Akaike Information Criterion & Bayesian Information Criterion \\
$(1,1)$ & -1112.76 & -1085.65 \\
$(1,2)$ & -1109.73 & -1078.11 \\
$(1,3)$ & -1124.78 & -1088.64 \\
$(2,1)$ & -1113.20 & -1081.58 \\
$(2,3)$ & -1139.57 & -1098.91 \\
$(2,4)$ & -1123.59 & -1078.41 \\
$(3,1)$ & -1113.39 & -1077.25 \\
$(3,3)$ & -1138.05 & -1092.87 \\
$(3,4)$ & -1121.83 & -1072.14 \\
$(3,5)$ & -1123.02 & -1068.81 \\
$(4,1)$ & -1130.89 & -1090.23 \\
$(4,3)$ & -1131.14 & -1081.44 \\
$(4,4)$ & $-\mathbf{1 1 4 0 . 4 4}$ & -1086.23 \\
$(5,1)$ & -1130.92 & -1081.22 \\
$(5,2)$ & -1130.92 & -1081.22 \\
$(5,3)$ & -1132.90 & -1078.69 \\
\hline \hline
\end{tabular}


Table 3: GARCH regression results: global mean temperatures

\begin{tabular}{lcccc}
\hline \hline variable & Regression 1 & Regression 2 & Regression 3 & Regression 4 \\
\hline$T_{t-1}$ & $-0.2598^{* *}$ & $-0.2691^{* *}$ & $-0.2655^{* *}$ & $-0.2675^{* *}$ \\
& $(0.0241)$ & $(0.0157)$ & $(0.0255)$ & $(0.0231)$ \\
$\ln \left(C_{t}\right)$ & $0.8758^{* *}$ & $0.9168^{* *}$ & $0.9016^{* *}$ & $0.8956^{* *}$ \\
& $(0.0933)$ & $(0.1017)$ & $(0.0892)$ & $(0.0888)$ \\
$C_{t}-C_{t-1}$ & - & 0.0087 & - & 0.0154 \\
& & $(0.0157)$ & & $(0.0138)$ \\
constant & $-1.785^{* *}$ & $-1.919^{* *}$ & $-1.872^{* *}$ & $-1.794 * *$ \\
& $(0.3716)$ & $(0.4127)$ & $(0.3588)$ & $(0.3590)$ \\
\hline \hline
\end{tabular}

Dependent variable: $T_{t}-T_{t-1}$

Standard errors in parentheses

$*$ : significant at $5 \%$ level

**: significant at $1 \%$ level 